\title{
The Problem of Scale in Electric Metrology of Nanostructures in the Context of the New SI Redefinition of the Base Electric Unit
}

\author{
W.T. ChYlA* \\ Applied Science Enterprise, P.O. Box 22, 00-975 Warszawa 12, Poland
}

(Received October 13, 2012)

\begin{abstract}
The classical, electrodynamic definition of the ampere is incoherent with quantum electrodynamics. The problem, although insignificant at the macroscopic scale, manifests clearly at the nanostructure level, where the consistently quantum approach is necessary. In this paper, we consider the Casimir effect to quantify inconsistencies that could have resulted if electric metrology of microstructures and nanostructures (including graphene) had been based on classical electrodynamics and the current SI definition of the ampere. The issue is discussed in the context of the New SI program, where the base electric unit is to be redefined by fixing the numerical value of the elementary charge. The conclusion supports the case for a prompt redefinition of the base electric unit, which will make the electric metrology in general, and the electric metrology of nanostructures in particular, coherent with the international system of units.
\end{abstract}

DOI: 10.12693 /APhysPolA.125.3

PACS: 06.20.fa, 85.85. + j, 85.35.-p, 31.30.jh, 06.20.Dk, 81.05.ue

\section{Introduction}

Current definitions of the SI base units reflect diverse points of view on how the base units ought to be defined; those views evolved considerably after the Metric Convention had been signed. The base unit of mass is still defined as the mass of the primary artefact (the International Prototype of the Kilogram); the kelvin is defined in terms of a physical property of bulk matter (the triple point of water); the second is defined according to Maxwell's concept of atomic standards (the caesium clock); the definition of the candela has evolved from the "established equations of science" (the black body radiation law); and the metre is defined by fixing the numerical value of a fundamental physical constant (the speed of light in vacuum, $c$ ) [1]. A variety of visions is a much celebrated value in humanities, but the diversity in primary definitions that establish the international system of units does not contribute to the coherence of the SI; this will change once the New SI is instituted, where all base units are defined in a uniform way, by fixing numerical values of physical constants [2].

The current definition of the ampere reads: "The ampere is that constant current which, if maintained in two straight parallel conductors of infinite length, of negligible circular cross-section, and placed 1 metre apart in vacuum, would produce between these conductors a force equal to $2 \times 10^{-7}$ newton per metre of length" [1]. Even in this single definition, one can recognize three different elements, characteristic of various methods of defining the SI units: (1) The electrodynamic definition of the ampere

\footnotetext{
*e-mail: chylawt@wp.pl
}

is based on "established equations of science" (Maxwell's equations); (2) it implicitly ${ }^{\dagger}$ fixes the numerical value of the magnetic constant $\mu_{0}$, which can be viewed as a precursor of the 1983 definition of the metre and a paradigm for the New SI concept; (3) the definition explicitly states the primary method of realization of the unit, which predetermines the mise en pratique of the ampere. If validity of any of the three elements is put into doubt, the whole definition can be questioned.

The above definition of the base electric unit had been worked out in the mid 1940's [3] and it was adopted by the 9th CGPM in 1948 [4]. The definition implicitly assumes that classical electrodynamics is perfectly exact. At nearly the same period of time (1946-1949) a new theory emerged, quantum electrodynamics ${ }^{\ddagger}$ (QED),

†Neither Maxwell's equations nor the magnetic constant are explicitly mentioned in the definition of the ampere, because the classical theory of the electromagnetic field had no alternative (QED) at the time when the definition of the ampere had been designed (mid 1940's). The numerical value of $\mu_{0}$ is implicit in the cited value of the electromagnetic force.

$\ddagger$ The QED has been preceded by (1) the old quantum theory of Planck, Einstein, Bohr, de Broglie and Compton, which assumed a phenomenological approach to quantization of the electromagnetic field and to motion of massive particles (1900-1925); and by (2) the canonical quantum theory of Heisenberg, Schrödinger and Dirac, where quantized massive particles interact with the classical electromagnetic field (1926-1945). The QED, the fully quantum theory of electrons and photons, developed by Dirac, Fermi, Bethe, Tomonaga, Schwinger and Feynman (1946-1949), has been followed by more general forms of the quantum field theory (QFT), such as the electroweak theory (Weinberg, Glashow, Salam, 1960s), quantum chromodynamics and the Standard Model (1960spresent), and the hypothetical Grand Unified Theory (1970spresent). Those advanced theories are not in the scope of metrology 
which showed that validity of Maxwell's equations is restricted to macroscopic situations, where the electromagnetic field does not manifest its quantum nature. Accordingly, the current definition of the ampere, which is based on the classical theory of the electromagnetic field, is suitable for macroscopic systems, but it is inadequate in the case of nanoscopic systems, where quantization of the electromagnetic field enters the picture.

Modern electric metrology works well because it has partly circumvented the problem of the discord between the classical definition of the ampere and the QED by switching to the primary quantum standards of voltage (based on the Josephson effect) and resistance (based on the quantum Hall effect), which are fully consistent with modern quantum physics [6]; however, the classical definition of the base electric unit remained unchanged. The move has been endorsed by the 19th CGPM in 1991 [7], but the price for it is the SI-incompatibility of electric measurements traceable to these two primary quantum standards. The New SI redefinition of the base electric unit ${ }^{\S}$ will remove the current dichotomy in electric units traceable to the SI ampere or to the primary quantum standards, and this is a priority for electric metrology, subjectively equally important and even more urgent than the redefinition of the SI kilogram "

Another, related reason for a prompt redefinition of the base electric unit, which is taken up in this paper, is the emergence of a new field of electric metrology, i.e. metrology of nanostructures, which preferably should be consistent with the international system of units from the very beginning. Electric measurements in the micro/nano scale might give significantly different results, where the raw measurement data (e.g. displacements in miniaturized micro-electromechanical system (MEMS) or nano-electromechanical system (NEMS) devices [8]) are interpreted with the use of the classical approximation or in the QED regime; the rule of thumb is that the differences increase where the size of the considered system decreases.

The SI definition of the ampere, based on Maxwell's equations, implies the possibility of current-scaling and size-scaling in primary realizations of the base electric unit, where maintaining of exactly 1 A currents in par-

yet, because metrology has not formally entered the high-energy domain so far. However, it is only a matter of time when the international metric system of units covers also the high-energy domain and the SI base units for the weak and the strong interactions will have to be designed [5].

$\S$ Although the proposed version of the New SI keeps the ampere as the base electric unit [2], there are strong physical and logical arguments for choosing the coulomb as the base unit.

\However, redefinition of the kilogram by fixing the numerical value of the Planck constant $h$ is a precondition for fully coherent incorporation of the quantum standards of voltage and resistance into the structure of the international system of units, because the Josephson constant and the von Klitzing constant involve both the elementary charge $e$ and the Planck constant $h$. allel conductors that are exactly $1 \mathrm{~m}$ apart is not feasible in practice (recall the current balance). The classical SI definition of the base electric unit does not allow for switching to more advanced theories of the electromagnetic field, consistent with the size of the considered system, because otherwise the definitional value of the unit would become dependent on the level of theoretical sophistication of the SI user. Consequently, modern electric measurements in nanoscopic systems are not consistently traceable to the classical definition of the ampere and classical realizations of electric units.

The Casimir effect provides an opportunity to illustrate quantitatively the problem at hand. In classical electrodynamics, the energy and the force of interaction between two flat, parallel, electrically neutral, conductive plates that carry no current are exactly zero. This is not so in quantum electrodynamics, where quantum fluctuations result in a nonzero energy and force of interaction between the two plates. The Casimir force increases rapidly with the decreasing separation between the plates. A comparison of the Casimir interaction with the classical interaction between surface currents or surface charges provides a measure of how significant quantum effects are in electric measurements in layered structures of a given size.

In this paper, we examine the size-scaling problem that concerns the SI-traceable electric measurements in layered structures; it involves the interplay of three elements: the Casimir effect, metrology of nanostructures, and the redefinition of the base electric unit. Due to the high symmetry of the considered system, the force and the energy of the Casimir interaction can be expressed in explicit, closed forms, which significantly simplifies the analysis. We begin with a brief review of the Casimir effect, sufficient for further considerations, and provide references to primary works and more advanced treatments. In the next section, we compare the Casimir interaction with classical electromagnetic interactions in layered structures, to quantify discrepancies between the classical and the quantum interpretation of raw electric measurements in layered structures of different size. The subsequent section gives a brief summary of the magnitude of quantum effects in electric measurements, related to typical values of electric quantities in layered structures; we draw on typical parameters that occur in those structures (including graphene), such as carrier density, current density, bias voltage, and strength of the electric field. The comparison shows that the problem is usually negligible in millimetre-size structures, the quantum effects are clearly noticeable in electric measurements of microstructures, and the classical approach fails entirely at the nanostructure level, as expected. This supports the call for a prompt redefinition of the base electric unit, as a precondition for making the electric metrology of nanostructures fully compatible with the international system of units. A brief general discussion concludes this paper. 


\section{Basics of the Casimir effect reviewed}

This section provides basic information on the origin of the Casimir effect, the Casimir force, energy of the Casimir interaction and the lifetime of quantum fluctuations that manifest as the Casimir effect; references are given to reviews and original works on the subject.

\subsection{Phenomenological intuition}

In the classical approximation, the physical vacuum is just an empty space. In the quantum approach, the physical vacuum hosts short-lived virtual particles, whose creation and annihilation complies with the Heisenberg uncertainty principle. The dominating component of vacuum fluctuations in the low-energy range are virtual photons and electron-positron pairs (the QED domain), whereas particle-antiparticle pairs of higher masses contribute to the observable processes only in the high-energy regime (the electroweak theory, the QCD and the standard model). In the low-energy domain, vacuum fluctuations are responsible for the Lamb shift, the anomalous value of the magnetic moment of the electron (both effects are important for scientific metrology), radiative corrections in scattering processes, photonphoton scattering, etc.

The Casimir effect is a manifestation of asymmetry between vacuum fluctuations in free space and in the volume subject to boundary conditions. Consider a system of two flat, parallel, perfectly conducting plates, separated by a gap of width $a$. The continuous density of states that are available to virtual photons in the two half-spaces beyond both plates, matches that of the infinite free space. The discrete density of states in the gap between the two plates is lower, because the boundary condition (vanishing of the electric field $\boldsymbol{E}_{\|}$on both boundary surfaces) implies that only certain eigenstates are available to virtual photons. The lower density of states within the gap accommodates a lower density of virtual photons, compared with the density of virtual photons in the two outer half-spaces"l. Consequently,

\| A similar effect occurs in the case of massive charged particles (carrier electrons or holes) in layered semiconductor nanostructures. The lower, discrete density of states in a thin layer (in the potential quantum well) is not able to accommodate as many carriers as the bulk matter can, which results in depletion of carriers in the thin layer. Consequently, the layer acquires the net electric charge, which affects the potential function for the nanostructure and (1) shifts the quasi-stationary eigenstates in the quantum well and the corresponding spectral lines [9]; (2) shifts the current resonances in the nanostructure [10]. Besides the analogy between the discrete carrier eigenstates and the discrete photon eigenstates in a thin layer, there are also important differences: (1) charge carriers are fermions and therefore must obey the Pauli exclusion principle, whereas photons are bosons that are not subject to such a constraint; (2) since the interaction of charged carriers is much stronger than the interaction of virtual photons, the canonical quantum theory is sufficient to handle the former case, whereas the Casimir effect is a subtle phenomenon that requires employment of more interaction of the two plates with the confined virtual photon field is weaker than the interaction with vacuum fluctuations outside the gap. The asymmetry of the interaction with vacuum fluctuations inside or outside the gap is the source of the net force (the Casimir force) that pushes the plates toward each other.

An equivalent phenomenological explanation of the origin of the Casimir effect emphasises the role of charge carriers inside the plates: fluctuations of the carrier density in any of the two conducting plates give rise to the short-lived local net charges, which induce local net charges of the opposite sign in the other plate. Interaction between the induced charges gives rise to the (usually attractive) Casimir effect. The two phenomenological explanations of the Casimir effect are equivalent, just as in the case of the classical description of a charged capacitor, where the energy of the interacting system can be viewed either as (1) a consequence of the electrostatic interaction between (real) charges of opposite signs on both plates; or equivalently (2) as a property of the electric field between both plates.

\subsection{Magnitude of the Casimir force}

The magnitude of the Casimir force $F$ (per unit surface $^{* *}$ ) of interaction between two flat, parallel, uncharged, perfectly conducting plates, depends strongly on the size of the gap $a$ between both plates [11]:

$$
F=\frac{\pi h c}{480 a^{4}} \text {. }
$$

Figure 1 shows the magnitude of the Casimir force (continuous line) as a function of the size of the gap, $a$. Since $a$ and $F$ can vary over many orders of magnitude, the logarithmic scale is used on both axes; the relationship $F(a)$ in the double logarithmic scale takes on the easy-to-read, linear form.

The size parameter $a$ of the layered structure is measured in nanometres, ranging from $a=1 \mathrm{~nm}=10 \AA$ $(\log a=0)$ to $a=10^{6} \mathrm{~nm}=1 \mathrm{~mm}(\log a=6)$. The corresponding Casimir force ranges from $F=1.3 \times$ $10^{-15} \mathrm{~N} / \mathrm{m}^{2}$ (for $a=1 \mathrm{~mm}$ ) to $F=1.3 \times 10^{9} \mathrm{~N} / \mathrm{m}^{2}$ (for $a=1 \mathrm{~nm}$ ). The graph also shows the Young modulus of graphene $\left(1.09 \times 10^{12} \mathrm{~N} / \mathrm{m}^{2}[12,13]\right)$, the tensile strength of graphene $\left(1.3 \times 10^{11} \mathrm{~N} / \mathrm{m}^{2}[14]\right)$, the stress that has been recorded in suspended multilayer graphene sheets $\left(1.5 \times 10^{9} \mathrm{~N} / \mathrm{m}^{2}[15]\right)$, and the atmospheric pressure of $1.013 \times 10^{5} \mathrm{~N} / \mathrm{m}^{2}$, just for comparison. It follows that the Casimir force is negligible in macroscopic structures $\left(a \geq 10^{5} \mathrm{~nm}\right)$, it is small but appreciable in microstructures $\left(10^{2} \mathrm{~nm} \leq a \leq 10^{5} \mathrm{~nm}\right)$, and can be very significant in nanostructures $\left(a \leq 10^{2} \mathrm{~nm}\right)$, reaching values $\sim 10^{4}$ times higher (at $a=1 \mathrm{~nm}$ ) than the atmospheric pressure.

sophisticated methods (although the full-blown QED formalism is not necessary).

** Since the Casimir force is given per unit surface, it is actually the Casimir pressure, which is a more precise but rarely used term. 


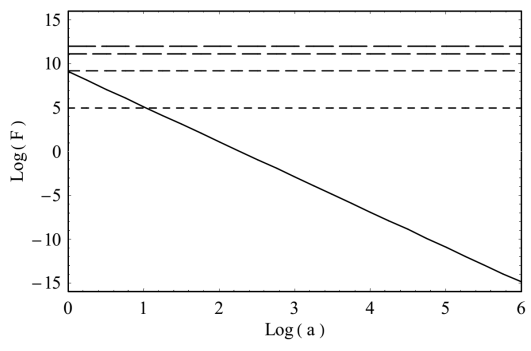

Fig. 1. Magnitude of the Casimir force $F$ per unit surface (continuous line) is shown as a function of the separation $a$ between both plates. The horizontal broken lines show respectively (from the top one): (1) the Young modulus of a monolayer graphene; (2) the tensile strength of graphene; (3) a typical stress that results from imperfections of the graphene lattice; and (4) the atmospheric pressure. The scale on both axes is logarithmic. The unit of $F$ is N/m $\mathrm{m}^{2}$ and the unit of $a$ is $\mathrm{nm}$.

\subsection{Magnitude of the Casimir energy}

The magnitude of the Casimir energy per unit surface, $E$ is [11]:

$$
E=\frac{\pi h c}{1440 a^{3}}
$$

and varies from $E=4.3 \times 10^{-19} \mathrm{~J} / \mathrm{m}^{2}$ (for $a=1 \mathrm{~mm}$ ) to $E=4.3 \times 10^{-1} \mathrm{~J} / \mathrm{m}^{2}$ (for $a=1 \mathrm{~nm}$ ). The Casimir force and the Casimir energy satisfy the usual relation between the force and the potential energy, $F=-\mathrm{d} E / \mathrm{d} a$. Figure 2 shows the Casimir energy as a function of the size of the gap, $E(a)$, see the continuous line; the logarithmic scale is used on both axes.

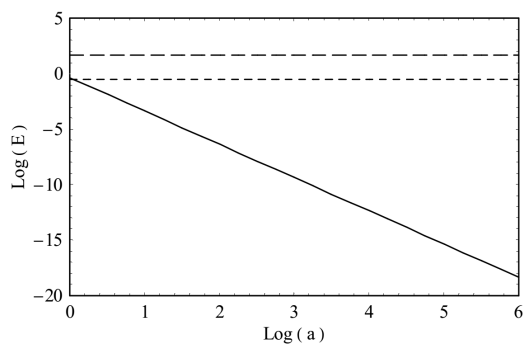

Fig. 2. Magnitude of the Casimir energy $E$ per unit surface (continuous line) is shown as a function of separation $a$ between both plates. The horizontal broken lines indicate respectively (from the top one): (1) the enthalpy of formation/atomization of graphene per $1 \mathrm{~m}^{2}$; and (2) the energy of the interlayer interaction in graphite. The scale on both axes is logarithmic. The unit of $E$ is $\mathrm{J} / \mathrm{m}^{2}$ and the unit of $a$ is $\mathrm{nm}$.

The enthalpy of graphene has not been measured yet. Assuming that the dissociation enthalpy of one $s p^{2} \mathrm{C}-\mathrm{C}$ aromatic bond of length $L=0.142 \mathrm{~nm}$ in graphene is ${ }^{\dagger \dagger}$ $\Delta H_{\mathrm{c}-\mathrm{c}}=4.87 \times 10^{5} \mathrm{~J} / \mathrm{mol}$, one gets the estimate of

\footnotetext{
${ }^{\dagger}$ Different sources give estimates of $\Delta H_{\mathrm{c}-\mathrm{c}}$ that vary from the
}

the enthalpy of formation/atomization of a monolayer graphene $^{\ddagger \ddagger} \Delta H_{\mathrm{g}}=46.5 \mathrm{~J} / \mathrm{m}^{2}$; this value and the energy of the interlayer interaction in graphite $\left(0.32 \mathrm{~J} / \mathrm{m}^{2}[17]\right)$ are shown in Fig. 2 for comparison, see the broken lines.

\subsection{Lifetime of the Casimir-type vacuum fluctuations}

The characteristic lifetime $\Delta t$ of vacuum fluctuations of energy $\Delta E=\pi h c /(1440 a)$ in the volume $a^{3}$ can be determined from the Heisenberg principle

$$
\Delta t \approx \frac{h}{4 \pi \Delta E} \approx \frac{36.5 a}{c} .
$$

In smaller volumes, energies of quantum fluctuations $\Delta E$ are larger, their lifetime $\Delta t$ is shorter and vice versa: larger volumes imply fluctuations of lower energy, which however last longer. For example, for $a=1 \mathrm{~nm}$ the energy of vacuum fluctuations is $\Delta E=4.3 \times 10^{-19} \mathrm{~J}=$ $2.7 \mathrm{eV}$ and their lifetime is $\Delta t=1.2 \times 10^{-16} \mathrm{~s}$, whereas for $a=1 \mu \mathrm{m}$, the corresponding quantities are $\Delta E=$ $4.3 \times 10^{-22} \mathrm{~J}=0.0027 \mathrm{eV}$ and $\Delta t=1.2 \times 10^{-13} \mathrm{~s}$.

Equation (3) shows that the lifetime of virtual photons is $\approx 36$ times larger than the travel time of light between the plates, which a posteriori justifies the phenomenological model where the discrete virtual photon eigenstates within the gap are represented by standing waves with nodes on both interfaces.

\subsection{Primary works and the contemporary research on the Casimir effect}

The first presentation of what is now called the Casimir effect was given in 1948 [18]. The work was a spin-off of his earlier research on interaction between a perfectly conducting plate and a polarizable particle [19]. The theoretical predictions of Casimir were first confirmed experimentally by Sparnaay in 1958 [20].

What had begun as a theoretical curiosity became a research subject of practical importance when miniaturization of electronic elements reached the sub-micrometre level; the topic is of growing interest in recent years due to the advent of graphene-based electronics, where active elements are of nanometre width. Relevance of the Casimir effect to practical problems in the electronics

low value of $4.52 \times 10^{5} \mathrm{~J} / \mathrm{mol}$ to the high value of $5.19 \times 10^{5} \mathrm{~J} / \mathrm{mol}$. The magnitude of $\Delta H_{\mathrm{c}-\mathrm{c}}$ varies, because the "strength" of the aromatic bond (bond energy) depends on the aromatic carbon skeleton of the reference molecule (benzene or fused aromatic rings) and substituents that affect density of delocalized electrons in the rings. We assumed the intermediate value of $\Delta H_{\mathrm{c}-\mathrm{c}}=4.87 \times 10^{5} \mathrm{~J} / \mathrm{mol}$ [16], which ensures that other estimates of $\Delta H_{\mathrm{c}-\mathrm{c}}$ lie within $\pm 8 \%$.

$\ddagger \ddagger$ The low average energy $E$ of the Casimir interaction (compared with the enthalpy of graphene $\Delta H_{\mathrm{g}}$ ) does not warrant that the virtual field cannot facilitate migration of impurities, vacancies, dislocations and other imperfections of the lattice, because of the statistical nature of quantum fluctuations. The problem of spontaneous migration, thermal migration and electromigration in graphene-based nanostructures is of practical importance, because properties of a monolayer structure are much more sensitive to imperfections of the lattice than the bulk material. 
industry has broadened the range of research topics in this field. The current research concentrates on studying the Casimir force with the use of different materials and thickness of the plates (metals, engineered metamaterials, and especially much attention is devoted to one or both plates made of graphene), where the gap can be filled with a dielectric material; investigations also concern different geometries of the plates, the edge effects, temperature dependence of the Casimir force, interaction of the conducting plate with carbon nanotubes, multilayer systems, repulsive Casimir force, and some topics of general interest, such as modification of the Lamb shift in a cavity, or a new limit on participation of massive particles in mediation of the gravitational interaction [21-34]. Since this paper employs the Casimir effect merely to examine a specific problem of quantum effects in electric metrology of nanostructures, in view of the classical definition of the ampere, we shall settle for this brief outline and refer the reader interested in the in-depth studies of the Casimir effect to reviews [35-41] that cover the subject comprehensively and provide abundance of relevant references.

\section{Magnitude of the Casimir-equivalent classical electric quantities}

Let us assume, just for the sake of the argument, that quantum standards of electric quantities are not used (as they are inconsistent with the SI ampere) and the raw data are interpreted in classical terms (just for consistency); then, the Casimir effect would have distorted the measurement results to a degree dependent on the size of the considered system ${ }^{\S}$. In this section, we exemplify the magnitude of this problem in the case of a few electric quantities.

\subsection{The Casimir-equivalent classical surface current density}

Let us compare the Casimir force $F$ with the classical electrodynamic force of interaction $F_{I}$ (see Eq. (A1)) between two planar, uniform surface current densities separated by a distance $a$. Having equated the two forces one gets the Casimir-equivalent, classical surface current density $I_{F}$ :

$$
I_{F}=\sqrt{\frac{\pi h c}{240 \mu_{0} a^{4}}},
$$

which is a measure of discrepancies one would encounter if measurements of the surface current density were (mis)interpreted by using classical electrodynamics and the SI definition of the ampere, in a structure that requires quantum treatment.

$\S \S$ Needless to say that metrology is a pragmatic science, which resorts to quantum standards and quantum theory whenever it is necessary, even at the price of departing from the definitional orthodoxy and coherence with the SI system of units.
We can also compare the Casimir energy $E$ with the classical energy of interaction between two planar, uniform surface current densities (see Eq. (A2)), thus obtaining the Casimir-equivalent, classical surface current density $I_{E}$ :

$$
I_{E}=\sqrt{\frac{\pi h c}{720 \mu_{0} a^{4}}},
$$

which also is a measure of discrepancies occurring if classical electrodynamics and the SI definition of the ampere were used in a situation where the QED enters the picture. As expected, the two quantities, $I_{F}$ and $I_{E}$, have the same dependence on the characteristic dimensional parameter $a$ and differ only by a numerical factor $\approx 1.7$. Thus, each of the two quantities can be used to gauge the "price" for using Maxwell's equations and the classical SI definition of the ampere in the quantum domain.

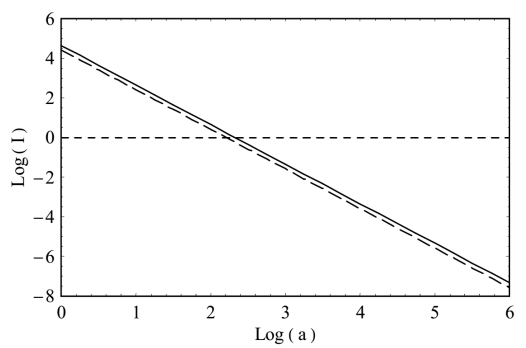

Fig. 3. The classical surface current density $I$ in two parallel current-carrying plates, which results in the force of interaction or the energy of interaction equal to the Casimir force or the Casimir energy ( $I_{F}$ continuous line or $I_{E}$ broken line, respectively), shown as a function of separation $a$ between both plates. The horizontal broken line represents a typical surface current density in graphene, $I_{0}=1 \mu \mathrm{A} / \mathrm{nm}$. The scale on both axes is logarithmic. The unit of $I$ is $\mu \mathrm{A} / \mathrm{nm}$ and the unit of $a$ is $\mathrm{nm}$.

The Casimir-equivalent, classical surface currents $I_{F}(a)$ and $I_{E}(a)$ are shown in Fig. 3. Magnitudes of the currents depend strongly on the scale of the structure and range from $I_{F}=4.5 \times 10^{-8} \mu \mathrm{A} / \mathrm{nm}$ and $I_{E}=2.6 \times 10^{-8} \mu \mathrm{A} / \mathrm{nm}$ for $a=1 \mathrm{~mm}(\log a=6)$ to $I_{F}=4.5 \times 10^{4} \mu \mathrm{A} / \mathrm{nm}$ and $I_{E}=2.6 \times 10^{4} \mu \mathrm{A} / \mathrm{nm}$ for $a=1 \mathrm{~nm}(\log a=0)$. Let us note that the real (physical) current density in (monolayer) graphene can reach "only बฯ" one to a few $\mu \mathrm{A} / \mathrm{nm}[44,45]$, and currents as high as $\sim 10^{4} \mu \mathrm{A} / \mathrm{nm}$ would damage the sample: the Casimir-equivalent current is a symbolic measure of how far we are off the track, if the quantum structure is analysed in classical terms, and it is not surprising that the

ฯ The current-carrying capacity of monolayer graphene, multilayer graphene, nanoribbons and nanotubes is $10^{11} \div 10^{13} \mathrm{~A} / \mathrm{m}^{2}$ [42-44] (the quantity is restricted by the Joule heat dissipation ability and electromigration), which is a few orders of magnitude higher than that of copper in printed circuit boards $\left(\approx 3 \times 10^{7} \mathrm{~A} / \mathrm{m}^{2}\right)$. 
Casimir-equivalent quantity might acquire values that exceed the physically realistic figures.

The ratio of the Casimir-equivalent current to the real (i.e. physical) surface current is a (relative) measure of discrepancy between the classical or quantum treatment of a given system: the smaller the ratio, the better for the classical approximation. In routine measurements, where the three-digit accuracy is sufficient, the ratio $\sim 10^{-3}$ is satisfactory; however, reliable reference standards require that the ratio is on the order of $10^{-8}$ or less. The lower values of the ratio are easier to attain where the real current is high; highest surface current densities in layered structures can be carried by graphene, where the typical quantity is $I_{0}=1 \mu \mathrm{A} / \mathrm{nm}$, although other carbon nanostructures, e.g. nanotubes, can carry current densities a few times larger. Figure 3 shows that if we stick with classical notions and definitions scaled down to the $a=1 \mathrm{~nm}$ level (e.g. the double layer of graphene), then measurements of the current with the use of NEMS could be off by over 4 orders of magnitude from the correct result. The systematic relative error associated with the Casimir interaction approaches $\sim 10^{-8}$ only for millimetre-sized structures $(\log a \approx 6)$.

\subsection{The Casimir-equivalent classical surface charge density}

We can also compare the Casimir force $F$ with the classical electrostatic force of interaction $F_{\sigma}$ (see Eq. (A3)) between two uniform surface charge densities $\sigma=Q / S$, where $Q$ is the magnitude of the electric charge on each of the surfaces $S$, separated by a distance $a$. Equating the two forces, we get the Casimir-equivalent, classical surface charge density $\sigma_{F}$ :

$$
\sigma_{F}=\sqrt{\frac{\pi h c \varepsilon_{0}}{240 a^{4}}} .
$$

Having compared the Casimir energy $E$ with the classical electrostatic energy of interaction $E_{\sigma}$ (see Eq. (A4)) between two uniform surface charge densities, we obtain the Casimir-equivalent, classical surface charge density $\sigma_{E}$ :

$$
\sigma_{E}=\sqrt{\frac{\pi h c \varepsilon_{0}}{720 a^{4}}} .
$$

The two Casimir-equivalent surface charge densities, $\sigma_{F}$ and $\sigma_{E}$, are shown in Fig. 4 as functions of $a$. The ratio of the Casimir-equivalent surface charge density to the real (i.e. physical) surface charge density in a double-layered structure is a (relative) measure of how significant quantum effects are in the static case. The smaller the ratio, the classical approximation works better and the scaling problem is less important; large real charge densities on the plates make the ratio smaller.

A layered structure can acquire the static electric charge due to natural depletion of carriers (due to a lower density of states in a layered structure, as compared with the bulk material) or due to the external potential that provides or drains carriers from the structure; the static charge density is therefore related to the carrier density. A typical carrier density in graphene at room temperatures is $\sim 10^{16} \mathrm{~m}^{-2}[46,47]$ (i.e. one carrier per $\approx 3.8 \times$
$10^{3}$ atoms of carbon), which implies that a typical surface charge density is $\sigma_{0}=10^{16} e_{0} / \mathrm{m}^{2}=1.6 \times 10^{-3} \mathrm{C} \mathrm{m}^{-2}$. The maximum observed carrier densities (both electrons or holes) in graphene are as high as $\approx 4 \times 10^{18} \mathrm{~m}^{-2}$ [48] (i.e. one carrier per $\sim 10$ atoms of carbon $^{* * *}$ ), which implies a possibility of reaching the surface charge density of $\sigma_{\max }=4 \times 10^{18} e_{0} / \mathrm{m}^{2}=0.64 \mathrm{C} \mathrm{m}^{-2}$.

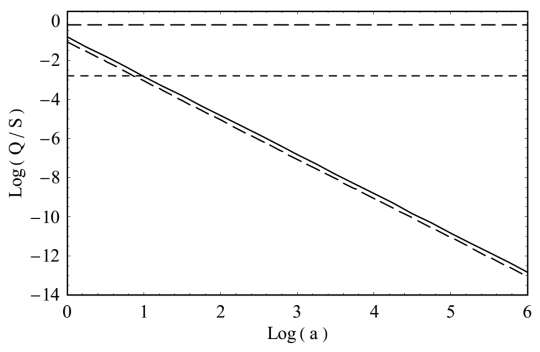

Fig. 4. The uniform surface charge density $\sigma=Q / S$ on two parallel plates, which results in the force or the energy of interaction equal to the Casimir force or the Casimir energy ( $\sigma_{F}$ continuous line or $\sigma_{E}$ broken line, respectively), shown as a function of separation $a$ between both plates. The horizontal broken lines indicate respectively (from the top): the high and a typical surface charge density in graphene. The unit of $\sigma$ is $\mathrm{C} / \mathrm{m}^{2}$ and the unit of $a$ is $\mathrm{nm}$.

Figure 4 shows that if we stick with classical electrodynamics and the present (classical) SI definition of the base electric unit, the static electric measurements could be off from the correct result by 2 orders of magnitude (at $a=1 \mathrm{~nm}$, in the case of a typical value $\sigma_{0}$ of the "real" surface charge density); the systematic relative error associated with the Casimir interaction decreases to $\sim 10^{-3}$ at $a$ on the order of a few hundred nanometers (microstructure level) and approaches $\sim 10^{-8}$ for $\sim 0.1$ millimetre-sized structures $(\log a \approx 5)$.

\subsection{The Casimir-equivalent classical voltage}

The Casimir force $F$ or the Casimir energy $E$ can be compared with the (classical) force $F_{U}$ (see Eq. (A5)) or the (classical) energy $E_{U}$ (see Eq. (A6)) of interaction between two parallel, flat, conducting surfaces of potential difference $U$. The comparisons yield the Casimir-equivalent, classical voltage $U_{F}$ :

$$
U_{F}=\sqrt{\frac{\pi h c}{240 \varepsilon_{0} a^{2}}}
$$

and $U_{E}$

$$
U_{E}=\sqrt{\frac{\pi h c}{720 \varepsilon_{0} a^{2}}},
$$

respectively, each of which can be interpreted as a measure of the magnitude of quantum fluctuations of voltage

\footnotetext{
***The high charge-storage capability of graphene makes it a prospective material for construction of powerful capacitors.
} 


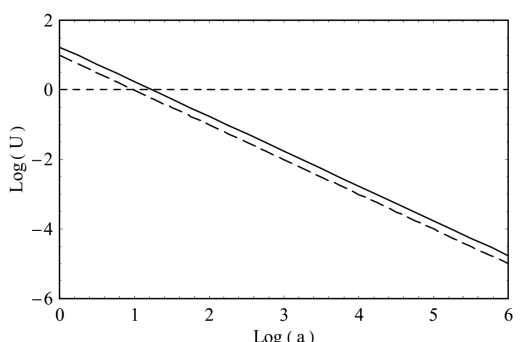

Fig. 5. The magnitude of the Casimir-equivalent, classical voltage $U$ between two parallel, flat, conducting surfaces, which results from the Casimir force $\left(U_{F}\right.$, continuous line) or the Casimir energy ( $U_{E}$, broken line), shown as a function of separation $a$ between both surfaces. The horizontal broken line shows the reference bias voltage $U_{0}=1 \mathrm{~V}$, which is typical in nanostructures. The units of $U$ and $a$ are $\mathrm{V}$ and $\mathrm{nm}$, respectively.

in the structure. The dependence of $U_{F}$ and $U_{E}$ on the size parameter $a$ is shown in Fig. 5 .

The ratio of the Casimir-equivalent, classical voltage to the external voltage actually applied to a layered structure quantifies the (relative) discrepancy that results from the classical approach in a situation where quantum fluctuations can occur. The lower the ratio, the better for validity of the classical approach.

A typical bias voltage in layered nanostructures is on the order of $U_{0}=1 \mathrm{~V}$, because resonant properties of nanostructures fade away at high biases (see e.g. Fig. 7 in [10]) and because problems with heat dissipation could result in increased temperature of the device, which facilitates electromigration; we shall assume $U_{0}$ as a constant reference quantity, although the bias voltage may be much higher in larger structures. Figure 5 shows that if the classical definitions and notions were scaled down to $1 \mathrm{~nm}$, it would have resulted in discrepancies as high as 1 order of magnitude; at $a \sim 10 \mu \mathrm{m}$ the discrepancy would have dropped to $10^{-3}$, and at $a \approx 1 \mathrm{~mm}$ the divergence would have been $\sim 10^{-5}$.

\subsection{The Casimir-equivalent classical electric field}

Having compared the Casimir force $F$ or the Casimir energy $E$ with the classical force $F_{E}$ (see Eq. (A7)) or the classical energy $E_{E}$ (see Eq. (A8)) of interaction between two parallel, flat, uniformly charged surfaces, one obtains the magnitude of the Casimir-equivalent, classical electric field $\boldsymbol{E}$ :

$$
\left|\boldsymbol{E}_{F}\right|=\sqrt{\frac{\pi h c}{240 \varepsilon_{0} a^{4}}}
$$

and

$$
\left|\boldsymbol{E}_{E}\right|=\sqrt{\frac{\pi h c}{720 \varepsilon_{0} a^{4}}}
$$

respectively. Since the time averaged value of quantum fluctuations of the electric field $\boldsymbol{E}$ is zero, the Casimir-equivalent field $|\boldsymbol{E}|$ should be understood as an estimate of the RMS value of the fluctuating field. The magnitude of the Casimir-equivalent electric field is shown in Fig. 6.

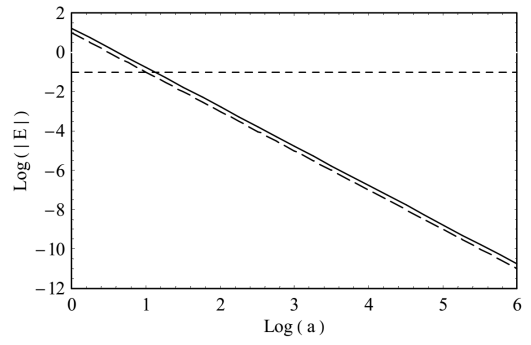

Fig. 6. The magnitude of the Casimir-equivalent classical electric field $|\boldsymbol{E}|$ between two parallel, flat, charged surfaces, which results from the Casimir force $\left(\left|\boldsymbol{E}_{F}\right|\right.$, continuous line) or the Casimir energy $\left(\left|\boldsymbol{E}_{E}\right|\right.$, broken line), shown as a function of separation $a$ between both surfaces. The horizontal broken line shows the reference value $E_{0}=0.1 \mathrm{~V} / \mathrm{nm}$, which is typical in nanostructures. The units of $|\boldsymbol{E}|$ and $a$ are $\mathrm{V} / \mathrm{nm}$ and $\mathrm{nm}$, respectively.

The ratio of the Casimir-equivalent, classical electric field to the strength of the real (i.e. physical) external electric field applied to a layered structure is a measure of the (relative) discrepancy associated with the classical treatment of such a system. The lower the ratio, the better for validity of the classical approach and for using the SI-traceable electric units.

For the sake of the argument, we shall assume that the external field strength is $\left|\boldsymbol{E}_{0}\right|=0.1 \mathrm{~V} / \mathrm{nm}$, which is typical in nanostructures. Figure 6 shows that quantum fluctuations of the electric field are higher than the typical external field for $a \leq 10 \mathrm{~nm}$; the ratio of the Casimir-equivalent field to the external field drops to $10^{-3}$ at $a \approx 0.4 \mu \mathrm{m}$ and falls further to $10^{-8}$ at $a \approx 0.1 \mathrm{~mm}$.

\section{Summary: the role of quantum fluctuations in the SI-traceable electric measurements in layered structures}

The Casimir-equivalent quantities are classical electric quantities that produce the same force or energy of interaction as the quantum Casimir effect ${ }^{\dagger \dagger \dagger}$. The Casimir-equivalent quantities gauge the magnitude of the QED effects in layered structures and provide a measure of the QED contributions to the systematic error - if the raw measurement data (e.g. displacements in electromechanical instruments) were interpreted in classical terms for the sake of coherence with classical realizations of the SI electric units. The Table summarizes the order of magnitude of these size-dependent, quantum contributions to the relative systematic error that would have occurred if one insisted on the consistently classical approach and the SI-traceability of electric measurements. 
Contribution of the Casimir effect to the relative systematic error in measurements of electric quantities in layered structures of various sizes, if the raw data were interpreted within the classical framework of the SI electric units.

\begin{tabular}{|c|c|c|c|c|c|}
\hline Quantity & $\begin{array}{l}\text { Reference } \\
\text { value }\end{array}$ & $\begin{array}{l}\text { Relative } \\
\text { systematic } \\
\text { error }\end{array}$ & $a=1 \mathrm{~nm}$ & $a=1 \mu \mathrm{m}$ & $a=1 \mathrm{~mm}$ \\
\hline $\begin{array}{l}\text { surface } \\
\text { current }\end{array}$ & $I_{0}=1 \mu \mathrm{A} / \mathrm{nm}$ & $\begin{array}{l}I_{F} / I_{0} \\
I_{E} / I_{0}\end{array}$ & $\begin{array}{l}4.5 \times 10^{4} \\
2.6 \times 10^{4}\end{array}$ & $\begin{array}{l}4.5 \times 10^{-2} \\
2.6 \times 10^{-2}\end{array}$ & $\begin{array}{l}4.5 \times 10^{-8} \\
2.6 \times 10^{-8}\end{array}$ \\
\hline $\begin{array}{l}\text { surface } \\
\text { charge }\end{array}$ & $\sigma_{0}=1.6 \times 10^{-3} \mathrm{C} \mathrm{m}^{-2}$ & $\begin{array}{l}\sigma_{F} / \sigma_{0} \\
\sigma_{E} / \sigma_{0}\end{array}$ & $\begin{array}{l}9.5 \times 10^{1} \\
5.5 \times 10^{1}\end{array}$ & $\begin{array}{l}9.5 \times 10^{-5} \\
5.5 \times 10^{-5}\end{array}$ & $\begin{array}{l}9.5 \times 10^{-11} \\
5.5 \times 10^{-11}\end{array}$ \\
\hline $\begin{array}{c}\text { bias } \\
\text { voltage }\end{array}$ & $U_{0}=1 \mathrm{~V}$ & $\begin{array}{l}U_{F} / U_{0} \\
U_{E} / U_{0}\end{array}$ & $\begin{array}{l}1.7 \times 10^{1} \\
1.0 \times 10^{1}\end{array}$ & $\begin{array}{l}1.7 \times 10^{-2} \\
1.0 \times 10^{-2}\end{array}$ & $\begin{array}{l}1.7 \times 10^{-5} \\
1.0 \times 10^{-5}\end{array}$ \\
\hline $\begin{array}{c}\text { RMS } \\
E \text {-field }\end{array}$ & $E_{0}=0.1 \mathrm{~V} / \mathrm{nm}$ & $\begin{array}{l}E_{F} / E_{0} \\
E_{E} / E_{0}\end{array}$ & $\begin{array}{l}1.7 \times 10^{2} \\
1.0 \times 10^{2}\end{array}$ & $\begin{array}{l}1.7 \times 10^{-4} \\
1.0 \times 10^{-4}\end{array}$ & $\begin{array}{l}1.7 \times 10^{-10} \\
1.0 \times 10^{-10}\end{array}$ \\
\hline
\end{tabular}

The reference values for the first two quantities (the surface current $I_{0}$ and the surface charge $\sigma_{0}$ ) are typical of graphene, whereas the reference values for the bias voltage $U_{0}$ (across the layered structure) and the electric field $E_{0}$ (in between the layers) are typical of "conventional" layered nanostructures. The relatively large contribution of quantum effects to the bias voltage in a macroscopic structure $\left(\sim 10^{-5}\right.$ for $\left.a=1 \mathrm{~mm}\right)$ is obviously the consequence of using the same reference value $U_{0}=1 \mathrm{~V}$ for all layered structures, whereas the typical voltage in macroscopic systems can be a few orders of magnitude higher (e.g. $10^{3} \div 10^{5} \mathrm{~V}$ in capacitors).

The magnitude of relative errors listed in Table validates neglecting the QED effects in analyses of measurement principles of macroscopic electromechanical instruments, whereas working principles of NEMS have to be analysed at the QED level, as expected. The general inference is that the classical SI definition of the ampere does not fit the needs of electric measurements in nanoscopic structures.

The intermediate range of the MEMS is a "grey area", where the situation has to be evaluated individually, depending on a particular device, the quantity to be measured and the required accuracy.

\section{Discussion and conclusion}

Maxwell's equations give a closed expression for the force of interaction between two straight, parallel wires of infinite length, separated by a distance $L$ in vacuum, which carry direct currents. If magnitudes of both currents are equal to $I$, the force $F$ exerted on a unit length of the wire is

$$
F=\frac{\mu_{0} I^{2}}{2 \pi L} .
$$

This expression is the basis for the 1948 definition of the ampere, which formally still stays in force: having assumed a certain value of the magnetic constant (permeability), $\mu_{0}=4 \pi \times 10^{-7} \mathrm{~V} \mathrm{~s} \mathrm{~A}^{-1} \mathrm{~m}^{-1}$, the unit of current is defined by the magnitude of the force of interaction that results from Eq. (12), $F=2 \times 10^{-7} \mathrm{~N} \mathrm{~m}^{-1}$. The definitional force $F$ scales as the square of the current $I$ and the inverse of the distance parameter $L$. However, in situations where quantum effects become significant, the scaling breaks down and the classical electromagnetic definition of the ampere fails. This causes the incoherence problem in the contemporary electric metrology: (1) If electric measurements are traceable to the SI units, the system fails at the nanoscale for fundamental physical reasons, and not just for insufficient accuracy or stability of classical realizations of the ampere or other electric units, as compared with quantum standards; (2) if the quantum approach and primary quantum standards are used, the measurements are not SI-traceable.

The classical electromagnetic definition of the ampere explicitly invokes a physical phenomenon, which (from the modern point of view) should actually be the domain of the mise en pratique of the unit, rather than occur in the definition. The magnetic constant $\mu_{0}$ is not explicitly mentioned in the classical definition of the ampere; its value is only implicitly assumed and associated with a certain (classical) theory of the electromagnetic field via the numerical value of the force of interaction that is cited in the definition. That approach makes the ampere dependent on the assumed theory of the electromagnetic field. With the advent of the QED, the magnitude of the base electric unit is not uniquely defined: the value of the ampere depends on the choice of the classical or quantum approach at a given scale of the considered system.

On the other hand, the New SI approach to defining base units is entirely different: the units are going to be defined by explicitly fixing the numerical values of physical constants, without any reference to physical theories and physical laws; those references are to be shifted to the mise en pratique of the units, i.e. to descriptions of the recommended methods for realization of the units. By fixing the value of a physical constant, we impose a constraint that determines one unknown, which - in this 
particular case - is the value of a unit; this new approach does not require making a prior commitment as to the kind of a relationship (i.e. the kind of a phenomenon or a physical law that is employed in the mise en pratique of the unit), which links the physical constant with the primary realization of the unit.

The choice of a physical constant that is employed to define a base unit is usually not unique. In the case of the base electric unit, one might define the ampere by explicitly fixing the value of the magnetic constant $\mu_{0}$, the electric constant $\varepsilon_{0}$, the traditional expression for the electromagnetic coupling constant $k_{E}=1 /\left(4 \pi \varepsilon_{0}\right)$, the fine structure constant $\alpha$, or the elementary charge $e$ [5]. The New SI ampere will be defined in terms of the elementary charge, because the fixed value of $e$ (together with the Planck constant $h$, fixed in the New SI definition of the kilogram) allows to determine the exact values of the von Klitzing constant $R_{\mathrm{K}}=h / e^{2}$ and the Josephson constant $K_{\mathrm{J}}=2 e / h$, that play the fundamental role in realization of electric units by means of the primary quantum standards of the ohm and the volt, respectively.

Redefinition of the base electric unit in terms of the fixed numerical value of the elementary charge will solve the problem of incompatibility of the classical definition of the ampere with quantum physics. The abstract definitions of the New SI base units are not only consistent with the present state of knowledge, but also immune to emergence of new physical theories and to the technological progress, because all references to physical laws, to specific physical phenomena, or to particular methods of realization of the (redefined) units will be shifted to their mises en pratique, which can be adjusted as necessary, without affecting the base definitions.

The electric metrology of nanostructures (carbon nanostructures in particular) has become especially important to the electronics industry in recent years. It is highly desirable that this emerging new field of metrology be developed in a situation free from bifurcation between the official, classical definition of the base electric unit and the laboratory practice, where the position of quantum standards is unchallenged.

Metrology has to keep pace with the needs of science and industry, and this calls for a prompt decision on the redefinition of the SI units. However, successful implementation of the New SI is contingent on: (1) achieving prior consensus on particular formulations of the New SI definitions of base units [49, 50]; and (2) gaining a widespread support for the reform, which requires much more information about the program directed not only to the professional measurement community, but also to other SI users and to the general public, i.e. taxpayers

$\ddagger \ddagger \ddagger$ The notable exceptions are the second (associated with a specific hyperfine transition spectral line) and the candela (associated with the black-body radiation law), which are not going to be redefined in the New SI; instead, definitions of these units will be rephrased. who will foot the bill for the upcoming changes in the international system of units. The annual World Metrology Day could provide an effective forum to publicize the idea of the New SI.

\section{Appendix: Interaction between surface currents or surface charges in classical electrodynamics}

Consider two flat, parallel, conducting plates, separated by a distance $a$. Let each of the plates carry the same uniform surface current density $I$. The force of interaction $F_{I}$ between the two currents is

$$
F_{I}=\frac{\mu_{0} I^{2}}{2}
$$

and the energy of interaction $E_{I}$ is

$$
E_{I}=\frac{a \mu_{0} I^{2}}{2} \text {. }
$$

If two flat, parallel plates carry the same uniform surface charge density $\sigma$, the force of the electrostatic interaction $F_{\sigma}$ between the two surface charges is

$$
F_{\sigma}=\frac{\sigma^{2}}{2 \varepsilon_{0}}
$$

and the energy of the interaction $E_{\sigma}$ is

$$
E_{\sigma}=\frac{a \sigma^{2}}{2 \varepsilon_{0}} \text {. }
$$

Let the potential difference between two flat, parallel plates be $U$. The force of the electrostatic interaction $F_{U}$ is

$$
F_{U}=\frac{\varepsilon_{0} U^{2}}{2 a^{2}}
$$

and the potential energy $E_{U}$ is

$$
E_{U}=\frac{\varepsilon_{0} U^{2}}{2 a},
$$

which can be expressed in terms of the uniform electric field $\boldsymbol{E}$ between the two plates as

$$
F_{E}=\frac{\varepsilon_{0} \boldsymbol{E}^{2}}{2}
$$

and

$$
E_{E}=\frac{a \varepsilon_{0} \boldsymbol{E}^{2}}{2},
$$

respectively. The relations $(\mathrm{A} 1)-(\mathrm{A} 8)$ give forces and energies per unit surface.

\section{References}

[1] The International System of Units, 8th ed., BIPM website, Sèvres 2006.

[2] I.M. Mills, Draft Chapter 2 for SI Brochure, following redefinitions of the base units, BIPM website, Sèvres 2010 .

[3] Resolution 2 of the CIPM in 1946, Definitions of electric units, BIPM website, Sèvres 1946.

[4] Resolution 6 of the 9th CGPM in 1948, Proposal for establishing a practical system of units of measurement, BIPM website, Sèvres 1948.

[5] W.T. Chyla, Acta Phys. Pol. A 120, 998 (2011). 
[6] C.J. Bordé, Philos. Trans. R. Soc. A 363, 2177 (2005).

[7] Resolution 2 of the 19th CGPM in 1991, The Josephson and quantum-Hall effects, BIPM website, Sèvres 1991.

[8] A. Bounouh, D. Bélières, Metrologia 48, 40 (2011).

[9] W.T. Chyla, W.D. Deering, Appl. Phys. A 55, 218 (1992).

[10] W.T. Chyla, W.D. Deering, Appl. Phys. A 58, 91 (1994).

[11] C. Itzykson, J.B. Zuber, Quantum Field Theory, McGraw-Hill, New York 1980, Sect. 3.2.4.

[12] C.D. Zeinalipour-Yazdi, C. Christofides, J. Appl. Phys. 106, 054318 (2009).

[13] C. Lee, X. Wei, J.W. Kysar, J. Hone, Science 321 385 (2008).

[14] T. Kuilla, S. Bhadra, D. Yao, N.H. Kim, S. Bose, J.H. Lee, Prog. Polym. Sci. 35, 1350 (2010).

[15] D. Garcia-Sanchez, A.M. van der Zande, A. San Paulo, B. Lassagne, P.L. McEuen, A. Bachtold, Nano Lett. 8, 1399 (2008).

[16] Short Handbook of Physico-Chemical Quantities, Eds. K.P. Mishchenko, A.A. Davidel, Chimia, Leningrad 1972 (in Russian).

[17] N.G. Chopra, L.X. Benedict, V.H. Crespi, M.L. Cohen, S.G. Louie, A. Zettl, Nature 377, 135 (1995).

[18] H.B.G. Casimir, Proc. Kgl. Ned. Akad. Wet. 60, 793 (1948).

[19] H.B.G. Casimir, D. Polder, Phys. Rev. 73, 360 (1948).

[20] M.J. Sparnaay, Physica 24, 751 (1958).

[21] G. Bressi, G. Carugno, R. Onofrio, G. Ruoso, Phys. Rev. Lett. 88, 041804 (2002).

[22] I. Brevik, S.Å. Ellingsen, K.A. Milton, Int. J. Mod. Phys. A 25, 2270 (2010).

[23] D. Drosdoff, L.M. Woods, Phys. Rev. B 82, 155459 (2010).

[24] M. Bordag, B. Geyer, G.L. Klimchitskaya, V.M. Mostepanenko, Phys. Rev. B 74, 205431 (2006).

[25] J. Sarabadani, A. Naji, R. Asgari, R. Podgornik, Phys. Rev. B 84, 155407 (2011).

[26] B.E. Sernelius, Europ. Phys. Lett. 95, 57003 (2011).

[27] J.N. Munday, F. Capasso, Int. J. Mod. Phys. A 25, 2252 (2010).

[28] O. Kenneth, I. Klich, A. Mann, M. Revzen, Phys. Rev. Lett. 89, 033001 (2002).
[29] N. Inui, K. Miura, e-J. Surf. Sci. Nanotechnol. 8, 57 (2010).

[30] D. Drosdoff, L.M. Woods, Phys. Rev. A 84, 062501 (2011).

[31] I.V. Fialkovsky, V.N. Marachevsky, D.V. Vassilevich Phys. Rev. B 84, 035446 (2011).

[32] V. Svetovoy, Z. Moktadir, M. Elwenspoek, H. Mizuta, Europ. Phys. Lett. 96, 14006 (2011).

[33] C.I. Sukenik, M.G. Boshier, D. Cho, V. Sandoghdar E.A. Hinds, Phys. Rev. Lett. 70, 560 (1993).

[34] R.S. Decca, D. López, E. Fischbach, G.L. Klimchitskaya, D.E. Krause, V.M. Mostepanenko, Phys. Rev. D 75, 077101 (2007).

[35] S.K. Lamoreaux, Phys. Today 60, (2) 40 (2007).

[36] M. Bordag, U. Mohideen, V.M. Mostepanenko, Phys. Rep. 353, 1 (2001).

[37] M. Bordag, G.L. Klimchitskaya, U. Mohideen, V.M. Mostepanenko, Advances in the Casimir Effect, International Series of Monographs on Physics, Vol. 145, Oxford University Press, Oxford 2009.

[38] A.W. Rodriguez, F. Capasso, S.G. Johnson, Nature Photonics 5, 211 (2011).

[39] K.A. Milton, The Casimir Effect: Physical Manifestations of Zero-point Energy, World Scientific, Singapore 2001.

[40] L. Pálová, P. Chandra, P. Coleman, Am. J. Phys. 77, 1055 (2009).

[41] S.K. Lamoreaux, Am. J. Phys. 67, 850 (1999).

[42] R. Murali, Y. Yang, K. Brenner, T. Beck, J.D. Meindl, Appl. Phys. Lett. 94, 243114 (2009).

[43] D. Kondo, S. Sato, K. Yagi, N. Harada, M. Sato, M. Nihei, N. Yokoyama, Appl. Phys. Expr. 3, 025102 (2010).

[44] A. Barreiro, M. Lazzeri, J. Moser, F. Mauri, A. Bachtold, Phys. Rev. Lett. 103, 076601 (2009).

[45] L. Liao, Y.C. Lin, M. Bao, R. Cheng, J. Bai, Y. Liu, Y. Qu, K.L. Wang, Y. Huang, X. Duan, Nature 467 (7313), 305 (2010).

[46] J.H. Chen, C. Jang, S. Xiao, M. Ishigami, M.S. Fuhrer, Nature Nanotechnol. 3, 206 (2008).

[47] S. Adam, E.H. Hwang, V.M. Galitski, S. Das Sarma, Proc. Natl. Acad. Sci. 104, 18392 (2007).

[48] D.K. Efetov, P. Kim, Phys. Rev. Lett. 105, 256805 (2010).

[49] W.T. Chyla, Metrologia 49, L17 (2012).

[50] W.T. Chyla, Metrologia 49, L11 (2012). 